\title{
Geographien des Ein- und Ausschlusses: Strafvollzug und -prozesse im Kontext der Aufarbeitung von Beteiligungshandlungen im syrischen Bürgerkrieg
}

\author{
Sarah Klosterkamp \\ Geographisches Institut der Universität Bonn, 53115 Bonn, Deutschland \\ Correspondence: Sarah Klosterkamp (s.klosterkamp@uni-bonn.de)
}

Received: 31 July 2020 - Revised: 23 April 2021 - Accepted: 23 April 2021 - Published: 10 May 2021

\begin{abstract}
Kurzfassung. Based on an ethnographic study of anti-terror trials at higher regional appeal courts in Germany, conducted in 2015-2020, this article examines the interrelation between the German penal system and criminal trials as mutually constitutive, governmentally guided, and highly secured elements of a state-induced and Islamcentred terrorism prevention. This includes the physical nature of the courthouses, as well as discourses of risk inscribed within them, which are linked to corresponding racialized and gender-rendered readings of the ,need for custody'. Under the auspices of a ,new penology ' and legitimized as an elimination of state-endangering actions", two logics emerge in the course of these proceedings that emphasize either a ,rectification of the reformable" or a ,confinement of the incorrigible', illustrating how a reshaped field of crime control and criminal justice can currently be observed that makes permanent incarceration the guarantor of a promise of security.
\end{abstract}

\section{Einleitung}

Spätestens seit Beginn der 2000er Jahre gab es nicht nur in Deutschland, sondern auch in seinen europäischen Nachbarländern Ausreisebewegungen von Menschen, die sich aufgrund einer antiimperialen Haltung, der Ablehnung, westlicher' Lebensweisen oder aber aus dem Wunsch nach einem politischen Systemwandel heraus in den Ländern des Mittleren und Nahen Ostens auch gewaltvoll für eine andere $\mathrm{Zu}$ kunft nach ihren Vorstellungen einsetzten und bereit waren, hierfür im Zweifel ihr Leben zu lassen. Sie taten dies zumeist an der Seite von Vereinigungen, die von ihren Herkunftsorten als ,islamistisch' klassifiziert und dementsprechend im Zuge von Antiterrormaßnahmen auch strafrechtlich verfolgt wurden. Doch erst 2014, mit der Gründung des sog. ,Islamischen Staates', den ersten Anschlägen auf europäischem Boden, einer Vielzahl an staatlich begleiteten oder induzierten Aus- und Einreisen im Spannungsfeld von Asyl und Flucht, wurde der deutschen Justiz bewusst, welch personell wie kapazitär vielschichtiges Problem auf sie zukommen würde. Dieses ,Problem', das in den Folgejahren medial wie politisch ins Zentrum der Debatte um die Frage nach geeigneten Resozialisierungs- und Integrationsmaßnahmen sowie der Notwendigkeit von Strafprozessen und -vollzug für eine gesamtgesellschaftliche Gefahrenminimierung rechtsstaatlicher Lesart rückte (vgl. Zöller, 2014; Graebsch, 2018), bildet den Ausgangspunkt dieses Beitrags.

Basierend auf einer Gerichtsethnographie während der Jahre 2015 bis 2020 sowie einer Foucault'schen Perspektive auf Strafprozesse zeige ich zum einen, auf welche Weise und mit welchen Mitteln die Verknüpfung staatlicher Gefahrenabwehr mit bestimmten Körpern, ihren intersektional verschränkten Lesarten und daraus abgeleiteten Gefahrenpotenzialen durch Gerichtsverfahren hervorgebracht, legitimiert und wirkmächtig wird. Zum anderen arbeite ich in Anlehnung an Garlands Unterscheidung zwischen einer ,Kriminologie des Selbst' vs. einer ,Kriminologie des Anderen ' die paradigmatische Bedeutung dieser Verfahren für die aktuelle Entwicklung und Transformation von Strafhaft heraus. Diese steht unter dem Signum von Sicherheit und ist seit einigen Jahren richtungsgebend für Art und Umfang des deutschen Strafvollzugs.

Hierfür werden zentrale Elemente der Transformation skizziert (Abschnitt 2) sowie daran anschließend die juridischen, disziplinaren und gouvernementalen Dimensionen staatlicher Gefahrenabwehr vorgestellt, die für das Rechts- 
subjekt ,Terrorist:in' konstitutiv sind: (1) die gesellschaftlichen Risikonarrative, die sie anleiten (Abschnitt 3.1), (2) die durch sie begründete sicherheitsbetonte Architektur deutscher Staatsschutzsäle (Abschnitt 3.2) und (3) die zur Haftprüfung, Verurteilung oder Bewährungsprüfung (vor-)geführten Körper und ihre normativ-diskursiven Lesarten (Abschnitt 3.3). Im Abschnitt 4 werden mittels eines Vergleichs die daraus resultierenden unterschiedlichen Bewertungslogiken einer ,Besserung der Besserungsfähigen “ (Abschnitt 4.1) vs. einer, Unschädlichmachung der Unverbesserlichen“ (Abschnitt 4.2) zur Diskussion gestellt. Diese Logiken münden in räumliche wie rechtliche, temporäre vs. dauerhafte Haft- und Externalisierungsweisen. Der Beitrag endet mit einem Fazit, in dem sein Argument noch einmal zusammengefasst präsentiert wird.

\section{Haft und Haftbedingungen im Spiegel staatlicher Gefahrenabwehr}

Wie Garland einst konstatierte, wird die Gesellschaft heute stärker denn je durch Verbrechenskontrolle und Strafjustiz bestimmt und folgt dabei eben jenen „Kulturen der Kontrolle" (Garland, 2008:74), die in ihrer punitiven Ausrichtung für die Verwahrung des Einzelnen besonders wirkmächtig sind. Beispielhaft für diese seit mehreren Jahrzehnten kontinuierlich fortschreitende Transformation der Sanktionierungsund Verwahrungsumstände sind eine präventionsorientierte Ausweitung polizeilicher Befugnisse sowie eine zunehmende Risikoorientierung im nationalen wie internationalen Strafrecht, welche die Implementierung einer Strategie der Kriminalitätsbekämpfung vorantreibt und konsolidiert, die sich nicht mehr (nur) auf die Abwehr konkreter Gefahren oder die Aufklärung von vorangegangenen Straftaten beschränkt, sondern zunehmend algorithmenbasierte Präventions- und Verwahrungsmechanismen weit im Vorfeld tatsächlich begangener Straftaten propagiert (vgl. Krasmann, 2006:234; Munk, 2017). Diese Entwicklung überschneidet sich mit ,westlichen “ Risikonarrativen einer ,globalized fear of terror" (Pain, 2009; Reuber und Strüver, 2012), und dies spätestens seit der Zunahme (a) von islamistischen Anschlägen in Europa und (b) den vermehrten Migrationsbewegungen aus den Krisen- und Konfliktregionen des Nahen Ostens. Sie lässt außenpolitische Belange und Fragen der inneren Sicherheit vermehrt unter xenophoben, antimuslimischen Vorzeichen zum Gegenstand weiterer Debatten und Sagbarkeiten im Feld der Kontrolle und Sicherheit werden (vgl. BTDrucksache 19/15668, 2019; Zöller, 2014).

Gerade in jüngerer Zeit warnen immer mehr Kolleg:innen der kritischen Geographie, Soziologie und Kriminologie vor den vielfältigen Gefahren und Auswirkungen solcher Tendenzen (vgl. Belina, 2016; Dübgen und Mattutat, 2017; Loick, 2018; Egbert und Krasmann, 2019; Keitzel, 2020). Dabei sind die Argumente, die dem aktuellen Trend polizeilicher Strafverfolgung und staatlicher Gefahrenabwehr zugrunde liegen, keineswegs neu. Im Zusammenhang mit den damit verbundenen, sicherheitsbegründeten bis profitorientierten Internierungstendenzen finden sie seit längerem noch drastischere Entsprechungen, gerade in angloamerikanischen Diskussions- und Gesellschaftsformationen (vgl. Rhodes, 2004; Gilmore, 2007; Turner, 2014; Dübgen und Mattutat, 2017; Herbert, 2019). Bemerkenswerterweise verweist auch die bundesdeutsche Debatte bei der Frage nach der Verhältnismäßigkeit der Sicherungsverwahrung bei der stetigen Zunahme ,dauerhaft verwahrungsbedürftiger' Körper (vgl. Abb. 1) auf ähnliche Begründungslogiken und Abwehrreflexe - trotz anderer Rechts- und Verwahrtradition. Auch hierzulande ist die Frage nach einer Ausweitung oder Eindämmung des Maßregelvollzugs ( $\$ 63$ bzw. $§ 64$ des Strafgesetzbuches - StGB) und seiner entmündigenden Tendenzen bis heute immer wieder Gegenstand öffentlicher wie wissenschaftlicher Debatten (vgl. Gericke und Kallert, 2007; Dübgen und Mattutat, 2017; Brazzell, 2018). Ihnen zugrunde liegen Einzelschicksale wie das von Gustl Mollat, der zu Unrecht sicherungsverwahrt wurde, oder die wiederkehrende Diskussion um einen angemessenen Umgang mit Sexualstraftäter:innen.

Während solche ambivalenten Zusammenhänge von Strafverfolgung und -vollzug vermehrt auch Eingang in die deutschsprachige Geographie gefunden haben - beispielsweise im Zusammenhang mit der Analyse von Haftumständen im Kontext lebenslanger Freiheitsstrafen Richter et al., 2017) sowie staats- und strafrechtlichen Dimensionen von Versicherheitlichung (Hannah, 2006; Belina, 2009; Korf, 2009) oder unter Thematisierung der komplexen Verschneidung von Armut als Ursache für Freiheitsentzug (Il-Tschung et al., 2017; Marquardt, 2019) -, stellte sich die gesellschaftliche Relevanz dieser vielfältigen Geographien des Ein- und Ausschlusses in den vergangenen Jahren nicht zuletzt auch vor dem Hintergrund der juristischen Aufarbeitung nationaler wie internationaler Beteiligungsformen im Kontext des syrischen Bürgerkrieges paradigmatisch zur Schau (Klosterkamp und Reuber, 2017). Paradigmatisch, weil hier die Feindfigur des, islamistischen Terroristen ' exakt jene Grenzziehung zwischen ,Regel' und ,Ausnahme', ,Innen “ und ,AuBen' markiert, die für die fortschreitende Transformation von Rechtsstaatlichkeit und Strafhaft unter dem Signum von Sicherheit bezeichnend ist. Dies betont auch Krasmann im Kontext des ,Feindstrafrechts‘ (vgl. Krasmann, 2006:235). Zwar gibt die Rechtsprechung im Zuge der Anwendung des neu eingeführten und ausgeweiteten $\S 129$ a des StGB sowie seine Erweiterung um Taten im Ausland durch Hinzunahme des internationalen Völkerstrafrechts (nach § 1 VStGB) vor, einen Ausschluss aus der Gesellschaft und eine Eliminierung von politischen Feinden als Ausnahme zu regeln. Aber es ist ,nicht die Ausnahme, die sich der Regel entzieht, es ist die Regel, die, indem sie sich aufhebt, der Ausnahme stattgibt" (Agamben, 2002:28) und entlang neoorientalistischer ,Freund'- vs. ,Feind'-Unterscheidungen durch das Recht ihre Legitimation erfährt (vgl. Krasmann, 2006:235; 


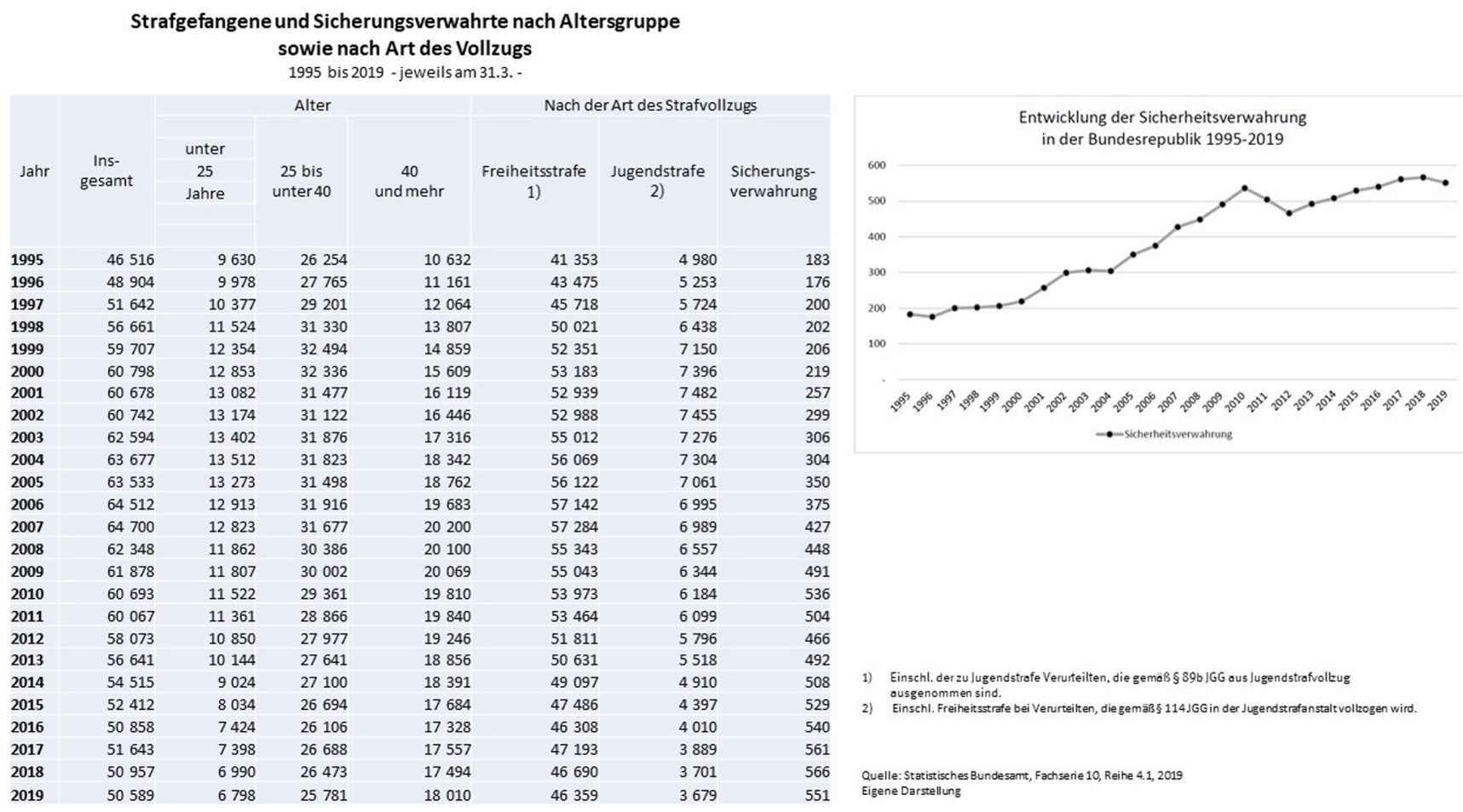

Abb. 1. Strafgefangene und Sicherheitsverwahrte nach Altersgruppe sowie nach Art des Vollzugs (1995-2019).

Klosterkamp, 2020; sowie zur rechtlichen Einordnung: Zöller, 2014:403; Schlepper, 2014).

Inwiefern eine solche Regelhaftigkeit bei der Abwehr, terroristischer Gefahrenlagen “ in der Bundesrepublik bereits eingetreten ist und in Anlehnung an Simon als „Governing through Crime“ (2007) beschrieben werden könnte, verdeutlicht die Zusammensetzung der Verwahrungsformen in deutschen Haftanstalten (vgl. Abb. 2). Während die Anzahl der inhaftierten Personen in der Tendenz insgesamt rückläufig ist, befindet sich rund ein Fünftel der Grundgesamtheit in Untersuchungshaft (ebd.; vgl. auch Dübgen und Mattutat, 2017:90). Ein überdurchschnittlich hoher Anteil der Untersuchungshaftinsass:innen sind dabei Angeklagte und hinreichend Verdächtige im Bereich der oben genannten Staatsschutzverfahren. Es sind gerade diese Verfahren, ihre besonderen Bestimmungen und die mit ihnen einhergehende überdurchschnittliche Verweildauer innerhalb der Haftanstalten, die den Rechtsstaat in den letzten Jahren in besonderer Weise herausforderten und trotz der Existenz unterschiedlicher Risiko- und Gefahrenkonjunkturen eine besondere Aufmerksamkeit erfuhren.

Mit der Zunahme der Fälle und ihrer öffentlichen Präsenz mehrten sich auch die Forderungen nach härteren Strafen und/oder aufenthaltsrechtlichen Konsequenzen (vgl. BTDrucksache 19/20201, 2020; Zöller, 2014). Dass diese Forderungen am Ende auch rechtswirksam vollzogen wurden, lässt sich an einer vergleichenden Analyse deutscher Staatsschutzverfahren aus den Jahren 2015 bis 2020 und den mit ihnen verbundenen Haft- und Abschiebemodalitäten nachzeichnen. So wurden im Rahmen eigener gerichtsethnographischer Arbeiten mehr als 26 Prozesse vor fünf Oberlandesgerichten (Düsseldorf, Stuttgart, Hamburg, München und Celle) und zwei Landgerichten (Hamburg und Köln) an mehr als 200 Präsenztagen teilnehmend beobachtet, protokolliert und im Hinblick auf ihre Anklageschriften, Verfahrensverläufe und Verurteilungen qualitativ ausgewertet ${ }^{1}$. Die Protokolle, die damit verbundenen Aktenbestandteile und die in der Erhebung triangulierten Beobachtungen ${ }^{2}$ sind die Datengrundlage, auf der die nachfolgenden Ausführungen basieren. Anhand ihrer zeige ich, dass die staatliche Gefahrenabwehr im Kontext des ,islamistischen Terrorismus' nicht zuletzt auch als Ausdruck einer rassifizierenden und vergeschlechtlichten Polizier- und Pönalisierungspraxis verstanden werden kann. Bei dieser Praxis wird in besonderer Weise die Anklage und temporäre Verwahrung vorrangig deklassierter, migrantisch-männlich gelesener Personen begünstigt.

\footnotetext{
${ }^{1}$ Für allgemeine Hinweise zum Projekt siehe auch: Klosterkamp und Reuber (2017), für eine methodische und forschungsethische Reflektion dieser Arbeit siehe: Faria et al. (2020), Klosterkamp (2020).

${ }^{2}$ Trianguliert wurden die Prozessbeobachtungen in den oben genannten Verfahren mit Aufenthalten in ethnographischen Nebenschauplätzen des Projektes, beispielsweise der Eröffnungsfeier am OLG Stuttgart, Einladungen in Anwaltskanzleien oder den vielen gemeinsamen Aufenthalten mit Familienangehörigen und anderweitig Assoziierten in den Wartebereichen der Gerichte.
} 


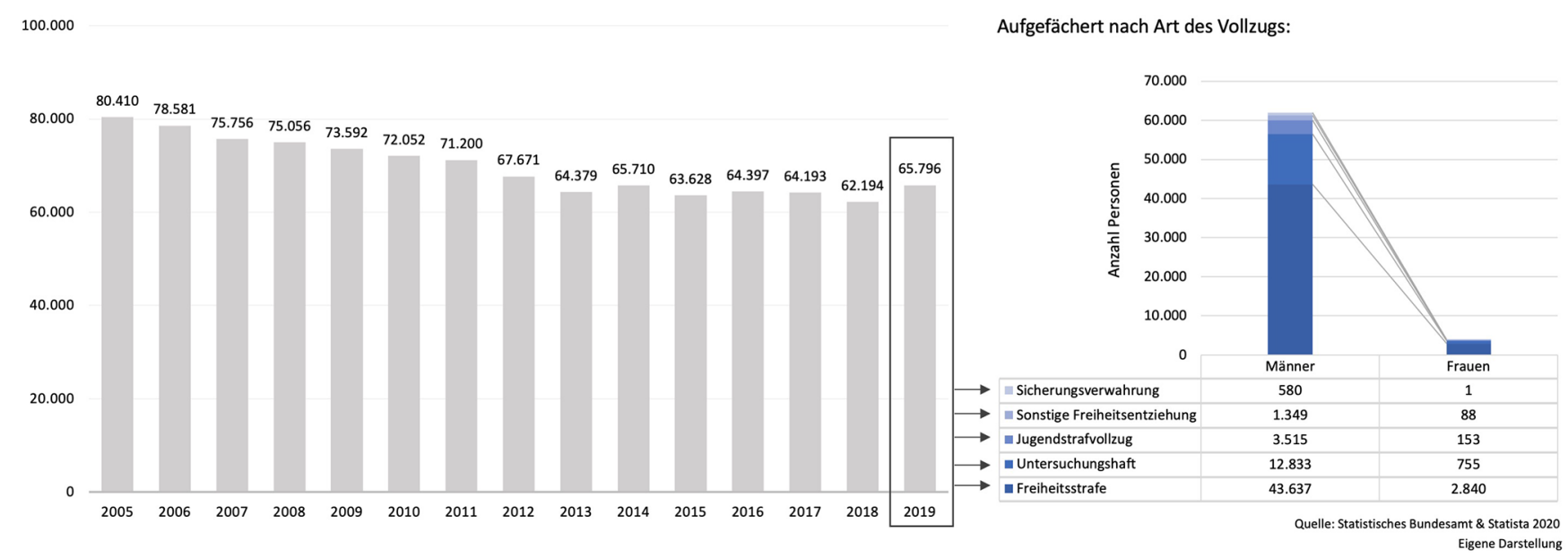

Abb. 2. Anzahl der Gefangenen und Verwahrten in den Justizvollzugsanstalten in Deutschland (2005-2019).

Dies ist die Folge eines komplexen Zusammenspiels aus konstatierter Notwendigkeit sowie multiplen Wirkungszusammenhängen juridischer, disziplinarer und gouvernementaler Macht und dadurch angeleiteter Formen des institutionellen Ein- und Ausschlusses (Abschnitt 3).

Zum anderen wird durch die Hinzunahme der Sicherungsverwahrung und der Möglichkeit asylrechtlicher (Folge-)Maßnahmen im Kontext dieser Verfahren eine Ausweitung der Haftdauer bzw. (national-)staatliche Ausgrenzungen sowohl ermöglicht als auch punitiv-praktisch vollzogen, wodurch nicht zuletzt das ,resozialisierbare Eigene ‘ vom ,bedrohlichen Anderen' auch mit und durch Haft klassifiziert und festgeschrieben wird (Abschnitt 4).

\section{Dimensionen staatlicher Gefahrenabwehr}

Wie im vorangegangenen Abschnitt aufgezeigt, verschiebt sich mit der strategischen Fokussierung auf die Vorwegnahme einer Gefahr häufig ihr Gegenstand - dies nicht zuletzt da, wo die Gefahr klassifiziert und eingehegt wird. Dies gilt in besonderer Weise für Institutionen des gesellschaftlichen Ein- und Ausschlusses, beispielsweise Gefängnisse und die ihnen vor- und nachgelagerten Rechtsinstanzen in Form von Gerichten, in denen die Aushandlung und Anrufung von (Un-)Freiheit und (Un-)Sicherheit gleichermaßen als Foucault'sche Instrumente einer ,Fremd'- und ,Selbstführung' gelesen werden können (Foucault, 1994 [1976]:16 f., 2004 [1977-1978]), die letztendlich als ,Dispositive der Macht` (Foucault, 1978:119 f.) zu einem Regieren und Einhegen pönalisierungsbedürftiger Rechtssubjekte führt (Simon, 2007). Dieses Wechselspiel aus juridischer, disziplinarer und gouvernementaler Macht wird aktuell vor allem im Bereich der juristischen Aufarbeitung in- wie ausländischer Beteiligungshandlungen im Kontext des Syrienkrieges offenkundig. So sind es neben den Risikonarrativen eines ,islamistischen Terrorismus' (Abschnitt 3.1), der besonde- ren Beschaffenheit der Arenen ihrer rechtsprechenden Praxis (Abschnitt 3.2) vor allem die in ihnen wirkmächtig werdenden polizeilichen Vorfeldermittlungen und damit einhergehenden Fokussierungen auf bestimmte, ,fremd ', , andersartig ' oder, ideologisch-fundamental ' gelesene Körper(-Praktiken) (Abschnitt 3.3), die entscheidend für die Sanktionierungsund Verwahrungslogiken der staatlich-induzierten Gefahrenabwehr sind, die aus ihnen hervorgehen.

\subsection{Die diskursiv-normative Dimension: Strafvollzug und Strafverfahren als ,notwendiges' Mittel im Kampf gegen den internationalen Terrorismus}

Handlungsleitend sowohl für die Art und Weise, wie Gerichtsgebäude im Bereich des Staatsschutzes ausgestaltet sind, als auch für die Frage, wer in diesen Arenen als, Gefahr für die innere und äußere Sicherheit' gelesen und angerufen wird, sind jene (Risiko-)Diskurse, die im Nachgang zu 9/11 eine besondere Wirkmächtigkeit entfaltet haben (vgl. Reuber und Strüver, 2012; Klosterkamp und Reuber, 2017). Als „globalized fear" (Pain, 2009:467) vor terroristischen Anschlägen finden sie dabei nicht nur Eingang in die Legitimationsschablonen internationaler Interventions- und Verteidigungskriege des globalen Westens, sondern werden als (geo)kulturell wie (geo-)politisch aufgeladene Sicherheits- und Risikonormierungen (vgl. Hannah, 2006; Korf, 2009) auch national-staatlich herangezogen, und zwar für die Errichtung und Inbetriebnahme entsprechender Sonderbauten. So heißt es in einer der ersten Kosten-Nutzen-Evaluationen der $\mathrm{Au}$ ßenstelle des Oberlandesgerichtes Düsseldorfs:

In der Vergangenheit sind wiederholt Stimmen laut geworden wie: Ist ein solch kostspieliges Prozessgebäude überhaupt erforderlich? Ist ein solcher Bau in Zeiten knapper Kassen überhaupt zu verantworten? Diese Fragen muss ich uneingeschränkt mit ,ja‘ beantworten. Die tragischen Ereignisse des 
11. September 2001 haben uns die immense Gefährdungslage globalen Ausmaßes klar und deutlich vor Augen geführt [... ]. Auch in diesen Fällen hat die Justiz die Aufgabe, den staatlichen Strafanspruch durchzusetzen. [... ]. Dazu gehört es aber auch, die Sicherheit der Prozessbeteiligten und der von diesen Verfahren betroffenen Bevölkerung zu garantieren. Diese Sicherheit kann es nicht zum Nulltarif geben (Paulsen, 2006:95).

Diese normativ-diskursiven Logiken der Herstellungsbedingungen von Sicherheit durch die umfassende Bekämpfung eines als ,islamistisch “ verstandenen Terrorismus im Inund Ausland wiederholen sich ständig. Sie werden nicht nur für die Legitimation besonders umfangreicher Steuerausgaben herangezogen, sondern sind auch das zentrale Begründungsmoment der staatlichen Einhegung derjenigen, die die Ordnung und Sicherheit zu gefährden drohen. Betont wird die Dringlichkeit ,einer konsequenten und effektiven Strafverfolgung etwaiger Anschläge im In- und Ausland“ (D6, 6. September 2016:4) $)^{3}$. Dabei wurde nicht zuletzt vor allem seine prominenteste Ausgeburt, der selbsternannte ,Islamische Staat' (IS), zur Zielscheibe und Orientierungsgröße für die Sanktionierungs-, Beurteilungs- und Verwahrungspraxis seiner Mitglieder und Sympathisant:innen. Quasi zur Standardeinleitung entsprechender Urteilsbegründungen der vergangenen fünf Jahre avancierte, dass der IS, ,wie keine andere Organisation in jüngster Zeit, dem internationalen Terrorismus ein neues Gesicht“ (D7, 23. November 2016:2) gegeben habe. Nun ist es genau diese Organisation, obwohl nur eine unter vielen, die nach den einstürzenden Türmen des World Trade Centers mit ihren wehenden schwarzen Fahnen, bewaffneten Menschen auf Pickup-Trucks und geladenen Maschinengewehren als prominenteste Referenz internationalen Terrorismus Einzug in die ,große Erzählung' nationaler Regulations-, Präventions- und Verteidigungsansinnen im Namen einer ,Gefahrenabwehr' hält, die auch ,,vor den eigenen Landesgrenzen keinen Halt machen $\mathrm{k}[\ddot{\mathrm{o}}] \mathrm{nn}] \mathrm{e} / \mathrm{und}$ d[ü]rf[e]“ (M2, 6. September 2016:69).

Die Vielfältigkeit seines Wirkens, die besondere Brutalität seiner Anschläge sowie die (juristische) Betonung der Gewissheit, dass neben etlichen ausländischen auch eine Vielzahl deutscher Staatsbürger:innen in seinen Reihen zu finden ist, deren länderübergreifende Vernetzung und zunehmende Rückkehr in ihre jeweiligen Ursprungsländer bereits seit längerem beobachtet und strafrechtlich verfolgt wird, sind gerade jene diskursiven Knotenpunkte, die Verfahren im Bereich des Staatsschutzes zu einer gesellschaftlichen Notwendigkeit werden lassen. Es geht um die Beteuerung, dass das Schädliche unschädlich gemacht, lange genug verwahrt, ab-

\footnotetext{
${ }^{3}$ Diese Referenz bezieht sich auf das dieser Publikation zugrunde liegende Datenmaterial unter Angabe der internen, hier anonymisierten Verweisstruktur mit Nennung von Datum und Seitenzahl des jeweiligen Transkripts bzw. Aktenbestandteils im Kontext der ausgewerteten Staatsschutzverfahren.
}

geschoben oder resozialisiert werde, um „Deutschlands Straßen, Weihnachtsmärkte und Konzertsäle jetzt und in Zukunft wieder zu sicheren, [zu] angstfreien Plätzen werden zu lassen“ (M3, 3. Juli 2018:14). Wie dieser Legitimationsrhetorik in Plädoyers und Anklageschriften der Exekutive ${ }^{4}$ entnommen werden kann, geht es immer auch um die Reifizierung der Notwendigkeit der Verfahren selbst, die mit jedem Urteil und jedem Vorzeigen und Benennen sanktionierungsbedürftiger Individuen ein Staatsschutzverfahren zu einem besonders wirksamen Instrument zur Herstellung nationaler Sicherheit werden lässt (D7, 23. November 2016:3 f.).

Die systematische Gesamtheit aus ,Bedingungen“, ,Ereignissen“ und ,Dingen“ definiert Foucault als „Archiv“ (Foucault, 1981 [1969]:186 f.). Das Archiv formiert für die Bedingungen von Aussagen einen Rahmen, der zu erklären hilft, warum Aussagen in aller Klarheit ausgesprochen, andere dagegen niemals explizit werden (vgl. ebd.). Als ,Katalysator des Sagbaren' besteht es im Bereich des Staatschutzes aus eben jener normativ-diskursiven Risikonormierung eines als ,islamistisch ' verstandenen ,Terrorismus “, der durch emotional-affektiv aufgeladene, (geo-)kulturelle und (geo-)politische Leit- und Feindbilder des ,Eigenen“ und des ,Fremden " und der Fokussierung auf sie bedienende Körper verstärkt wird. Durch das spezifische Zusammenspiel von disziplinierender Architektur und Subjektanrufungen wirken diese Horizonte des Sag- und Erwartbaren in den Arenen richterlicher Wahrheitssuche zum anderen auch an der KoKonstitution des Rechtssubjekts ,Terrorist' mit. Hier kumulieren sie und leiten so die dauerhafte Verwahrung der einen und eine zumindest für möglich erachtete Resozialisierung der anderen als staatliche Einhegung ,abstrakter' Gefahren an (vgl. Simon und Feeley, 1992:452; Simon, 2007).

\section{2 ,Form follows function': Die baulich-materielle Dimension von Staatsschutzprozessen}

Diese Einhegung wird durch gesellschaftliche Institutionen des Ein- und Ausschlusses materialisiert. Zu ihnen gehören die Justizvollzugsanstalten und die mit ihnen legitimatorisch wie prozessual eng verwobenen Strafprozessgebäude des Staatsschutzes. Zumeist angesiedelt an den Rändern der Stadt sowie versehen mit Hubschrauberlandeplätzen, verschiedenen Ein- und Ausfahrtsschneisen, Kontroll- und Wachposten sowie meterhohen, mehrfachreihigen Fluchtund Zugangsbarrieren, eint beide Gebäudearten eine sicherheitsbetonte Architektur, die sich gerade in den letzten Jahren vermehrt auf die (temporäre) Verwahrung und Verbringung hinreichend Verdächtiger solcher Terrorismusverfahren sowie der Gewährleistung der damit in Verbindung stehenden verschärften Sicherheitsregularien auszurichten hatte (vgl. Abb. 3, Bild 1). Aufgrund der Fülle an Verfahren und

\footnotetext{
${ }^{4}$ Hier ausgeführt durch den Generalbundesanwalt, der polizeiliche Ermittlungen im Bereich des Staatsschutzes anleitet und vor den Staatsschutzsenaten Klage führt.
} 

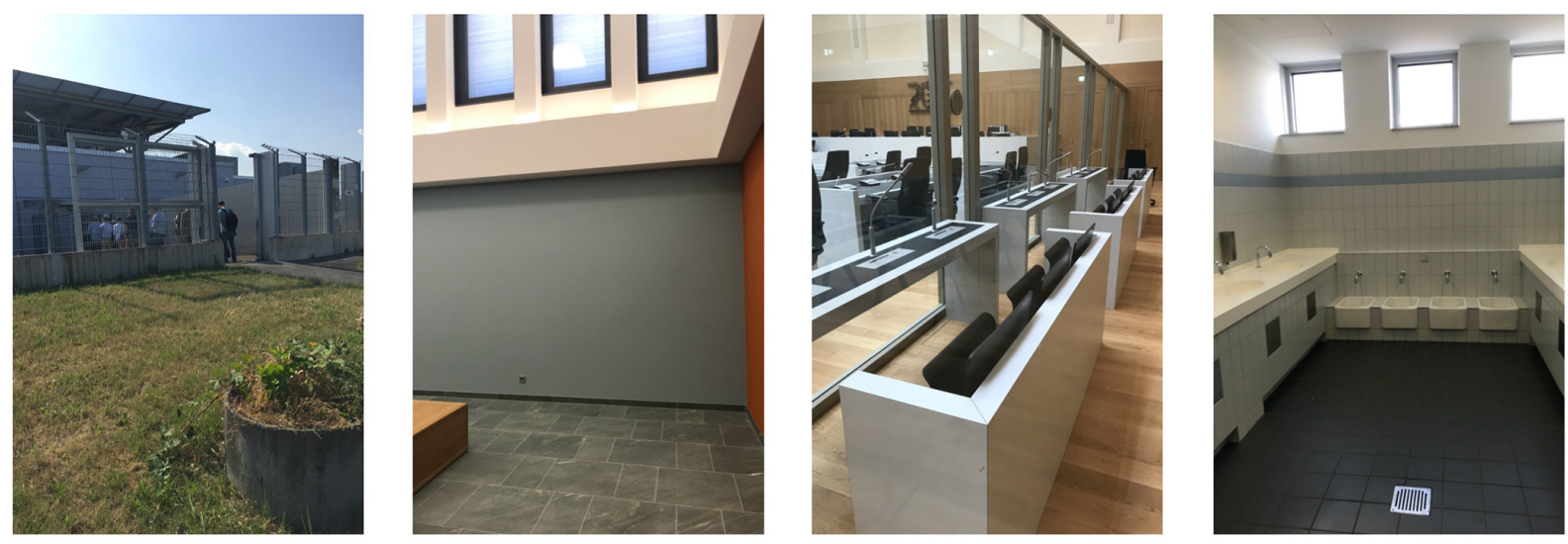

(c) Klosterkamp 2017, 2019, 2018, 2019

Abb. 3. Sicherheit und Architektur - Staatsschutzgebäude in Deutschland. Hier: das Oberlandesgericht Düsseldorf (links und rechts) sowie das Oberlandesgericht Stuttgart-Stammheim (mittig).

dem daraus resultierenden Umstand, dass nur wenige Haftanstalten diese Sicherheitsbestimmungen (inkl. der gesonderten Umschluss-, Transport- und Besuchsregularien) für die in der Regel mehr als ein Jahr andauernde Untersuchungshaft erfüllen (können), müssen viele der Untersuchungshaftinsass:innen mehrmals die Woche umverlegt oder zu den jeweiligen Prozessgebäuden gesondert transportiert werden sei es für das eigene Gerichtsverfahren, laufende Haftprüfungsentscheidungen oder weil sie im Zuge einer Aussage in anderen Verfahren als Zeug:innen (vor-)geführt werden.

Wie Alex Jeffrey betont, sind es dabei nicht nur die öffentlich-sichtbaren Insignien der Kontrolle und Überwachung, sondern auch eine "series of unseen infrastructures“ (Jeffrey, 2019:565), die den besonderen Charakter staatlicher Straf- und Verwahrungsprozeduren und ihre machtgeladene, rechtsprechende Praxis begleiten, unterstützen und verstärken. Wirkmächtig und erfahrbar wird dies durch ein Zusammenspiel aus Gepäck- und Personenkontrolle beim Einlass, durch ein von verbautem Spiegelglas und Kameras evoziertes Gefühl permanenter Überwachung sowie ganz allgemein einer Omnipräsenz der ,technology that facilitates [...] the recording of ambient noise in the wider court building, and the silencing effect of the securitized plastic dock" (ebd.) im Saalinneren, die in besonderer Weise zur Selbst- und Fremdführung der Anwesenden sowie der zur Verhandlung vorgeführten ,delinquenten Anderen' beitragen (vgl. Abb. 3, Bild 2+3). Während gerade der letzte Umstand, das Panzerglas, in deutschen Staatschutzprozessgebäuden die Angeklagten in mehrfacher Hinsicht von dem Rest des Saals abtrennt, sie abschirmt und als solches von Organen der Rechtspflege immer wieder als besonderes Hindernis und Wesensmerkmal dieser Verfahren hervorgehoben wird (D4, 3. Februar 2016:1; D7, 5. Oktober 2017), können dieser Aufzählung aus der Vielzahl der Prozesse weitere Aspekte hinzugefügt werden, in denen sich die Zurschaustellung staatlicher Macht auch materiell-baulich mani- festiert und die den Prozess richterlicher, Wahrheitsfindung und Sanktionierung ko-konstitutiv begleiten und anleiten: So sei neben der Höhe und Ausgestaltung etwaiger Bepolsterungen, Arm- und Rückenlehnen sowie das An- und Ablegen von Hand- und Fußfesseln (nach richterlicher Anordnung) auch auf symbolisch-funktionale Kleidungs- und Ausrüstungsgegenstände verwiesen (beispielsweise Schlagstöcke und Sicherheitswesten für Bedienstete sowie der Einlass mit Schreibunterlagen, Getränk und Elektronik nur für Journalist:innen, nicht aber für Angehörige), in denen sich die vorgegebenen Rollenzuweisungen und damit verbundenen (De-)Privilegierungen auch physisch bis in den Gerichtssaal hinein pausen. Sie dienen im Sinne einer „Macht der milden Mittel“ (Foucault, [1976] 1994:173 f.) öffentlichkeitswirksam als Subjektivierungs- und Distinktionsvorlagen für „Staat‘ vs. ,Delinquent:in“ im Verhandlungssaal sowie ,Vertrauten “ vs. ,Misstrauten' im Besucherraum.

Die Gebäude sind allerdings nicht nur in ihrer inneren, sondern auch in ihrer äußeren Funktion der materialisierte Ausnahmezustand einer "Geographie der Angst" (Korf, 2009:152). Sowohl hinsichtlich ihrer architektonischen Ausgestaltung als auch bei der Frage nach dem ,Wo' ihrer Errichtung werden verhaltenssteuernde Aspekte einer zu disziplinierenden Bevölkerung baulich berücksichtigt und in der tagtäglichen Ausführung bedient. So hieß es beispielsweise bei der Eröffnungsfeier des Prozessgebäudes am Oberlandesgericht Stuttgart-Stammheim im Mai 2019, dass nicht zuletzt deswegen für einen Neubau in unmittelbarer Nähe zur gleichnamigen Justizvollzugsanstalt entschieden worden sei, weil ,allein diese Stadtrandlage [...] davon ab[halte], dass allzu viel Protest, allzu viel Demonstrationen, allzu viel Auflauf da sind, das ist ja ganz anders, wenn man die Verfahren in unserem Stammhaus in der Innenstadt [durchführe]" (M5, 29. Juni 2019:5). Was die physische Distanz zur Stadt für die gouvernementale Sicherung nach Außen bewirken soll, verspricht man sich auch für das Innere des Gebäudes, 
und zwar durch die funktionale Trennung der Personengruppen und Zugangsbereiche:

Das zugrunde liegende Konzept war völlige Veränderung der öffentlichen und nicht-öffentlichen Bereiche. Das heißt, in der Regel kommt KEINER aus dem öffentlichen Bereich in den nichtöffentlichen Bereich. Das sind einfach Sicherheitsüberlegungen. Deswegen auch diese Wand [deutet auf das Panzerglas]. Im Altbau war da nichts. Das heißt, wenn da Skandale befürchtet wurden, musste man mit zehn, fünfzehn Wachmeistern den Bereich von Zuschauern und Gericht absichern. Heute kann diese Gefahr unter Umständen selbst bei sicherheitsrelevanten Verfahren mit ein oder zwei Wachmeistern verfolgt werden (ebd.).

Während sich damit die beiden größten bundesdeutschen Standorte Düsseldorf und Stuttgart in ihrer Foucault'schen Ausrichtung eines hierarchisch wie funktionell aufgeteilten, panoptischen Disziplinarraums (Foucault, 1994 [1976]: 183, 187, 191, 251 ff., 2004 [1977-1978]:76) grundlegend ähneln, die das Ziel der Minimierung von Unordnung verfolgt, eint beide Gebäude noch ein weiteres Element dieser sicherheitsbedachten wie Sicherheit hervorbringenden Form staatlicher Rechtsprechung. Denn obwohl diese hochgesicherten Prozesssäle prinzipiell für Verfahren gegen alle Formen von staatlich definiertem Terrorismus Anwendung finden, wurde in ihrer älteren, aber auch jüngeren Ausgestaltung wohl vor allem an ein dezidiert muslimisch-praktizierendes und genuin ,männliches ' Publikum gedacht. So sind auf den Herrentoiletten sowohl in dem Düsseldorfer Prozessgebäude von 2004 als auch im Stammheimer Neubau Gelegenheiten für rituelle Fußwaschungen verbaut (vgl. Abb. 3, Bild 4). Sie veranschaulichen, wie sich stereotype Zuschreibungen nicht zuletzt bis in die architektonische Ausgestaltung der Sanitärräume pausen. Bei der teilnehmenden Beobachtung und während gemeinsamer Pausenaufenthalte kommentierten dies die vorrangig muslimisch-praktizierenden weiblichen Besucherinnen unter Verweis auf das Fehlen von Pendants in den Damentoiletten und der funktionalen Trennung zwischen ritueller Waschung und Sanitäranlagen zumeist als „,widersprüchlich“ (D10, 22. Dezember 2016:1), gar ,zynisch“ (D10, 18. Januar 2017:21) oder als ,gut gemeint, aber fehlplatziert“ (D4, 25. Mai 2016:1). Viele von ihnen verwiesen zudem darauf, dass eine adäquate Ausrichtung für Gebete im Wartebereich nur erschwert möglich sei. Der Wartebereich sei einzig für Publikumsverkehr zugänglich, aber nicht mit Sichtelementen ausgestattet (D4, 6. April 2017). Hier fühlten sie sich für das Abhalten von Gebeten ohnehin durch Kameras und andere Prozessbeobachter:innen unnötig beobachtet bis gestört (D4, 16. Februar 2017). Sie machten ferner deutlich, dass sie das (partielle) Platzieren ritueller Waschgelegenheiten nicht zuletzt auch deshalb als (weitere) Form institutioneller Rassifizierung ansähen, weil nach ihren Erfahrungen solche Vorrichtungen in allen anderen staat- lich finanzierten Gebäuden Deutschlands mit muslimischem Publikumsverkehr gänzlich unbeachtet blieben (D12, 8. Juli 2018), beispielsweise in Schulen oder Asylunterkünften.

Die Zusammenschau dieser Alltags- und Erfahrungswelten sowie die dichte Beschreibung der auf unterschiedlichen Ebenen wirkmächtigen, panoptischen Risikonormierungen und -einhegungen ermöglicht, ,[...] an understanding of the dynamism of architecture, not as a static form but as a series of processes that enmesh the material, corporeal and affective“ (Jeffrey, 2019:570). Diese produktiven ,Mechanismen der Macht“ bezeichnet Foucault als „Mikrophysik“ (vgl. Foucault, 1994 [1976]:38, 40, 178, 191, 207). Sie begründen - wie im Folgenden verdeutlicht werden soll - nicht zuletzt auch die unterschiedlichen (gouvernementalen) Herstellungsweisen von Subjektkonstitutionen in und durch diese Verfahren. Sie sind in der Form von „Mikropraktiken“ (ebd.) allgegenwärtig, die - gemeinsam mit den genannten Risikodiskursen - die Strafverfolgung der hier Angeklagten und Verurteilten anleiten.

\section{3 ,Die Körper der Verurteilten“: Zur rassifizierten und vergeschlechtlichten Dimension staatlicher Gefahrenabwehr}

So wie die antizipierte Zuschauer:innenschaft in den Verfahren ist auch die für eine Verurteilung zu überprüfende Person in der Regel eine ,männliche " und vorwiegend ,muslimischmigrantisch“ gelesene. Während dieser Umstand zum einen der generellen Tendenz deutscher Haft- und Verwahranstalten entspricht, in denen überproportional mehr Männer als Frauen sowie mehr deklassierte als privilegierte Menschen freiheitsentziehend verwahrt werden (vgl. Abb. 2; Il-Tschung et al., 2017; sowie für den Zusammenhang von Geschlecht und Strafvollzug: Rodriguez et al., 2006; Schliehe, 2014), verdeutlicht er auch im Hinblick auf die mit ihm verbundenen Techniken des Polizierens das, was Singelnstein als „Confirmation Bias“ (Singelnstein, 2016:830) für die Arbeit deutscher Strafverfolgungsbehörden und ihre gerichtsrelevanten ,Outcomes' konstatiert: So würden Gerichte und Staatsanwaltschaften zu einem großen Teil vermehrt eben jene Personengruppen mit Haft- und Bewährungsstrafen belegen, gegen die im Zuge von ,Rasterfahndungen“ gezielt und überproportional stark ermittelt worden sei, gepaart mit einem ,Preventive Policing', angeleitet durch polizeiliche Kontroll- und Risikoanalysen (ebd.). Ein Auffinden und Verurteilen potenzieller ,Normen- und Regelbrecher' verstärke dabei diese Tendenzen, die sich dann als Bestätigungsfehler auf andere Verfahren desselben Delinquenzbereichs auswirken und hierdurch eine rassifizierende und klassenbezogene Strafverfolgungspraxis sowohl ermöglichen als auch dezidiert verstärken (Singelnstein, 2016:832; sowie für Preventive Policing: Belina, 2016; Egbert und Krasmann, 2019).

Genau diese Tendenz zeichnet sich in besonderer Weise auch im Bereich des Staatsschutzes ab. Wie auch Krasmann betont, werden hier am Ende ,nicht bestimmte Per- 


\begin{tabular}{|c|c|c|c|c|}
\hline \multicolumn{4}{|c|}{ Angeklagte nach $\S 129 a / b$, $\S 89$ StGB } & \multirow[t]{2}{*}{2015} \\
\hline Gesamt: & 33 & \multicolumn{2}{|c|}{ Staatsangehörigkeit(en): } & \\
\hline Männer & 32 & Deutsch* & 26 & \\
\hline Frauen & 1 & Andere & 7 & \\
\hline \multicolumn{4}{|c|}{ Angeklagt nach §129a/b, §89 StGB } & 2016 \\
\hline Gesamt: & 18 & \multicolumn{3}{|c|}{ Staatsangehörigkeit(en) } \\
\hline Männer & 17 & Deutsch* & 14 & \\
\hline Frauen & 1 & Andere & 4 & \\
\hline \multicolumn{4}{|c|}{ Angeklagte nach $\S 129 a / b$, $\S 89 \mathrm{StGB}$} & 2017 \\
\hline Gesamt: & 27 & \multicolumn{3}{|c|}{ Staatsangehörigkeit(en) } \\
\hline Männer & 27 & Deutsch* & 5 & \\
\hline Frauen & 0 & Andere & 22 & \\
\hline \multicolumn{4}{|c|}{ Angeklagte nach $\S 129 a / b, \S 89$ StGB } & 2018 \\
\hline Gesamt: & 16 & \multicolumn{3}{|c|}{ Staatsangehörigkeit(en) } \\
\hline Männer & 14 & Deutsch* & 6 & \\
\hline Frauen & 2 & Andere & 10 & \\
\hline \multicolumn{4}{|c|}{ Angeklagte nach $\S 129 a / b$, $\S 89$ StGB } & 2019 \\
\hline Gesamt: & 22 & \multicolumn{3}{|c|}{ Staatsangehörigkeit(en) } \\
\hline Männer & 13 & Deutsch* & 10 & \\
\hline Frauen & 9 & Andere & 12 & \\
\hline
\end{tabular}

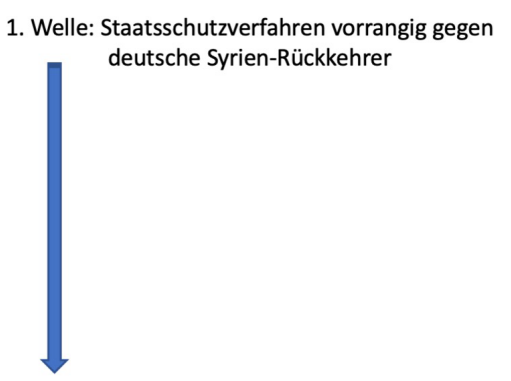

2. Welle: Staatsschutzverfahren vorrangig gegen syrische Geflüchtete

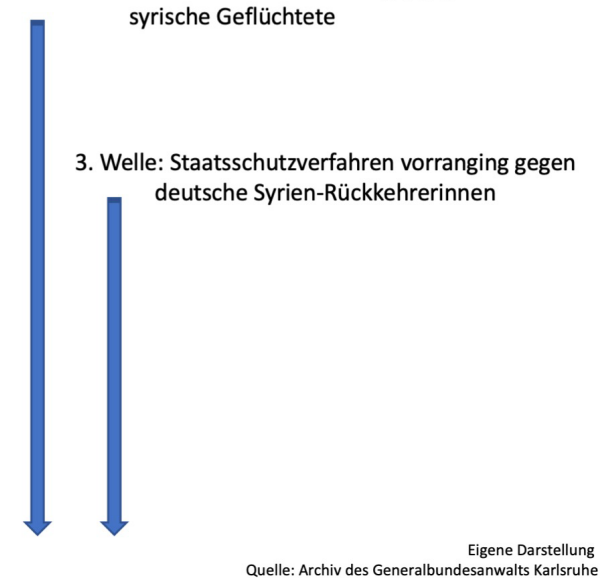

Abb. 4. Wellen deutscher Staatsschutzverfahren vor dem Hintergrund deutscher und ausländischer Beteiligungshandlungen im Kontext des syrischen Bürgerkrieges (2015-2020).

sonen [...] gesucht, sondern Typen“ (Krasmann, 2006:240). Ermöglicht und verstärkt wird diese Entwicklung durch die Rechtsauffassung selbst, die den Verfahren zugrunde liegt: Während Jurist:innen wie Zöller betonen, dass es in Deutschland ,keinen Sonderstrafrahmen für Terroristen [gibt]“ (Zöller, 2014:403), gelten die sog. Vereinigungsdelikte der $\S \S 129 \mathrm{a}+\mathrm{b}$ StGB ,,als Paradebeispiel für Strafnormen, die abstrakt gefährliche Verhaltensweisen im Vorfeld von organisierter und terroristisch motivierter Kriminalität unter Strafe stellen“ (ebd.:406). Pönalisiert werden bestimmte Mitwirkungshandlungen in Bezug auf kriminelle sowie terroristische Vereinigungen - im Inland und im Ausland. Wie Zöller dabei hervorhebt, würden die jeweiligen Individuen in diesem Kontext allerdings nicht bestraft, weil sie als Teil einer Gruppierung Straftaten tatsächlich begangen hätten, sondern kriminalisiert werden stattdessen Verhaltensweisen ,wie die Gründung, die mitgliedschaftliche Beteiligung, die Unterstützung und das Werben um Mitglieder und Unterstützer für eine Vereinigung, deren Tätigkeit auf die Begehung von Straftaten gerichtet ist. Ob diese Gruppierung bislang überhaupt schon Straftaten begangen hat oder jemals begehen wird, spielt dabei keine Rolle“ (ebd.).

Relevant für die strafrechtliche Verfolgung und somit für die entsprechende (Sicherungs-)Verwahrung ist somit nicht ausschließlich die Tat selbst, sondern vielmehr eine Ge- mengelage aus behördlich abgeleitetem Bedrohungspotenzial und konstatiertem Handlungsbedarf. Dies geschieht spätestens seit der Ausweitung dieser Verfahren um Taten syrischer Staatsbürger:innen nicht unter ,objektiven', sondern vor allem dezidiert geschlechtsbasierten sowie rassifizierten Vorzeichen: Während von der Vielzahl der aus Deutschland nach Syrien Ausgereisten rund $25 \%$ weiblich und die meisten von ihnen deutsche Staatsangehörige sind (vgl. BTDrucksache 19/20201, 2020:2), erschienen nur die wenigsten trotz eigener, teils längerfristiger und ebenso relevanter Beteiligungshandlungen als Angeklagte, sondern lediglich als Zeug:innen in den entsprechenden Staatsschutzverfahren. So sind von den bislang mehr als 2461 laufenden Strafverfahren im Kontext des syrischen Bürgerkrieges mehr als 2275 Verfahren gegen Personen mit einer anderen als der ausschließlich deutschen Staatbürger:innenschaft gerichtet, von denen sich lediglich 67 Verfahren auf weibliche Beschuldigte beziehen (vgl. ebd.; BT-Drucksache 19/6684, 2018:4 f.). Mit Blick auf diese Zahlen lässt sich konstatieren: Staatsgefährdender Terrorismus islamistischer Lesart ist aus Sicht von Strafverfolgungsbehörden vor allem ein ,männliches “ und ,von außen' importiertes Phänomen, wobei die damit zusammenhängende Ermittlungsarbeit vorwiegend rassifizierten und geschlechtsbezogenen Logiken $\mathrm{zu}$ folgen scheint (vgl. auch: Klosterkamp, 2020:6). 
Als Resultat dieser Ermittlungen lassen sich für die Jahre 2015 bis 2020 insgesamt drei Wellen von Anklagen im Bereich des Staatsschutzes identifizieren, die die vorliegenden Strafverfolgungstendenzen sowie -divergenzen pointiert zusammenfassen (vgl. Abb. 4). Ich unterteile sie nach Jahr, Geschlecht sowie Nationalität der jeweiligen Angeklagten: Für den Zeitraum 2015 bis 2016 liegt ein Schwerpunkt der Strafverfolgung auf männlichen deutschen*5 SyrienRückkehrern. Ab 2017 lässt sich eine zweite Welle an Verfahren feststellen, die vor allem mit der Verschärfung des Konfliktgeschehens in Syrien und damit vermehrten Fluchtund Migrationsbewegungen aus Syrien, dem Irak sowie Afghanistan in Verbindung zu bringen ist. Zugleich und bei rückläufiger Tendenz von Anklagen gegen männliche deutsche* Staatsangehörige kam es in den Jahren 2018 und 2019 $\mathrm{zu}$ einem politischen Richtungswechsel und somit zu einer vermehrten Strafverfolgung auch deutscher* Frauen, die im Nachgang eigener Syrienaufenthalte nun ebenfalls angeklagt und verurteilt wurden. Sie bilden somit eine dritte, wenn auch deutlich geringere Welle innerhalb dieser Typisierung, die in der Tendenz aber bis heute weiter ansteigt (vgl. BTDrucksache 19/11907, 2019).

Alle Angeklagten eint vor allem die Erfahrung, als Mitglieder und/oder Unterstützer:innen von Vereinigungen gelesen zu werden, die als ,terroristisch“ klassifiziert werden. Dies hatte entsprechende Ermittlungen, Durchsuchungsmaßnahmen und Vernehmungen zur Folge. Jedoch unterscheiden sich die Beschuldigtenvorgänge und Verwahrungstendenzen zugleich ganz grundsätzlich in der Art und Weise, inwiefern ihre Körper in den anschließenden Gerichtsprozessen als prinzipiell ,resozialisierbar' und ,mündig ' gelesen und welchen eben jene Potenziale in und durch diese Verfahren abgesprochen wurden.

\section{Haftbestimmungen und Hafttypologien im Bereich der Terrorismusabwehr als Vorzeichen einer New Penology?}

Im vorangegangenen Abschnitt wurden unter Anleitung einer Foucault'schen Perspektive auf Strafverfahren die zentralen Dimensionen staatlicher Disziplinierung skizziert, die sich für den Staatsschutz, seiner ihn anleitenden Risikodiskurse, Bauweisen und hier vorgeführten Köper vorwiegend als Antwort auf eine als ,männlich ‘ und ,islamistisch“ imaginierte Bedrohungslage formierten. Nun soll im Rückgriff auf Garland (2008) und der damit verbundenen Typenbildung entlang empirischer Beispiele aufgezeigt werden, wie sich in und durch Gerichtsverfahren verschiedene Momente einer ,Kriminologie des Selbst“ vs. einer ,Kriminologie der Anderen' (Garland, 2008:253) entfalten und durch Haft zueinander ins Verhältnis setzen. Während die eine beschworen wird, ,,um Kriminalität zur Routine zu machen, um über-

\footnotetext{
${ }^{5}$,deutsch*' meint hier das Vorliegen der deutschen und ggfs. einer weiteren Staatsbürgerschaft.
}

triebene Ängste abzubauen und präventives Handeln zu fördern, [...] dient [die andere] dazu, den Kriminellen zu dämonisieren, die in der Bevölkerung verbreiteten Ängste und Ressentiments auszuagieren und Unterstützung für staatliche Strafmaßnahmen zu erhalten“ (ebd). Der durch die Verfahren hergestellte Zuschnitt auf die intersektional unterschiedlich positionierten (Rechts-)Subjekte führt dabei aktuell zu zwei unterschiedlichen Logiken der Verwahrung. Sie werden im Folgenden als ,Besserung der Besserungsfähigen ‘ bzw. ,Verwahrung der Unverbesserlichen' bezeichnet. Aufgrund dieser Logiken sind die Urteile damit mehr für die Gesellschaft und mehr als die Sanktionierung von Verbrechen: Mit ihnen wird der Bereich des Staatsschutzes verlassen und eine staatlich induzierte Bevölkerungspolitik angeleitet, die die ,Besserungsfähigen“ von den ,Schädlichen“ sowohl identitätspolitisch wirksam klassifiziert als auch regulierend aussortiert.

\section{1 ,Besserung der Besserungsfähigen` - Zur Relationalität von Haft und Haftumständen}

Recht ist weder statisch noch persistent, sondern wandelbar und stets abhängig von gewissen Zeitlichkeiten und den Gegenständen, auf die es angewandt werden soll. So unterliegt auch die Frage, ,wer' oder,was' als Beteiligung an einer ,terroristischen Vereinigung ' gemäß deutscher Rechtsprechung gilt und einer freiheitsentziehenden Verwahrung und Sanktionierungsmaßnahme bedarf, zeitlichen Schwankungen und (Dis-)Kontinuitäten. Richtungsweisend für die Strafverfolgung von Organisationen, die als ,terroristische Vereinigungen' klassifiziert werden, ist in Deutschland das Ministerium für Justiz und Verbraucherschutz, das entsprechende Strafverfolgungsermächtigungen ausgibt, aktualisiert und/oder, in selteneren Fällen, verwirft. Durchgesetzt und als Recht wirksam werden diese Strafverfolgungsermächtigungen in den Arenen richterlicher Praxis. Hier fanden gerade in den vergangenen Jahren und im Kontext Syrien mitgliedschaftliche Beteiligungshandlungen und Unterstützungsleistungen sowohl für den ,Islamischen Staat im Irak und Großsyrien` (ISIG/IS) als auch für die Vereinigungen Anwendung, die mit ihm teils kooperierten, teils rivalisierten. Juristisch bewertet und bestraft wurden dabei nicht nur Ausreisen und Aufenthalte ins Ausland, sondern auch erfolgreich ausgeführte oder vereitelte Anschläge im Namen der Vereinigungen im deutschen Inland (vgl. beispielsweise BTDrucksache 19/20201, 2020:2 f.).

Eine Auswertung von mehr als 26 solcher Verfahren 6 unter Zuhilfenahme der ihnen zugrunde liegenden Ankla-

\footnotetext{
${ }^{6}$ Angeklagt wurden in den 26 Verfahren zusammengenommen insgesamt 46 Personen - 10 Frauen und 36 Männer deutscher wie ausländischer Staatsbürgerschaft. Die Fallauswahl ist damit fast erschöpfend in Bezug zur Gesamtanzahl aller bislang durchgeführten Verfahren der Jahre 2015 bis 2020, die sich auf insgesamt 33 Staatsschutzprozesse gegen 55 Personen beziffern lässt (vgl. beispielsweise BT-Drucksache 19/5648, 2018:15 sowie das Onlinearchiv des Generalbundesanwalts).
} 


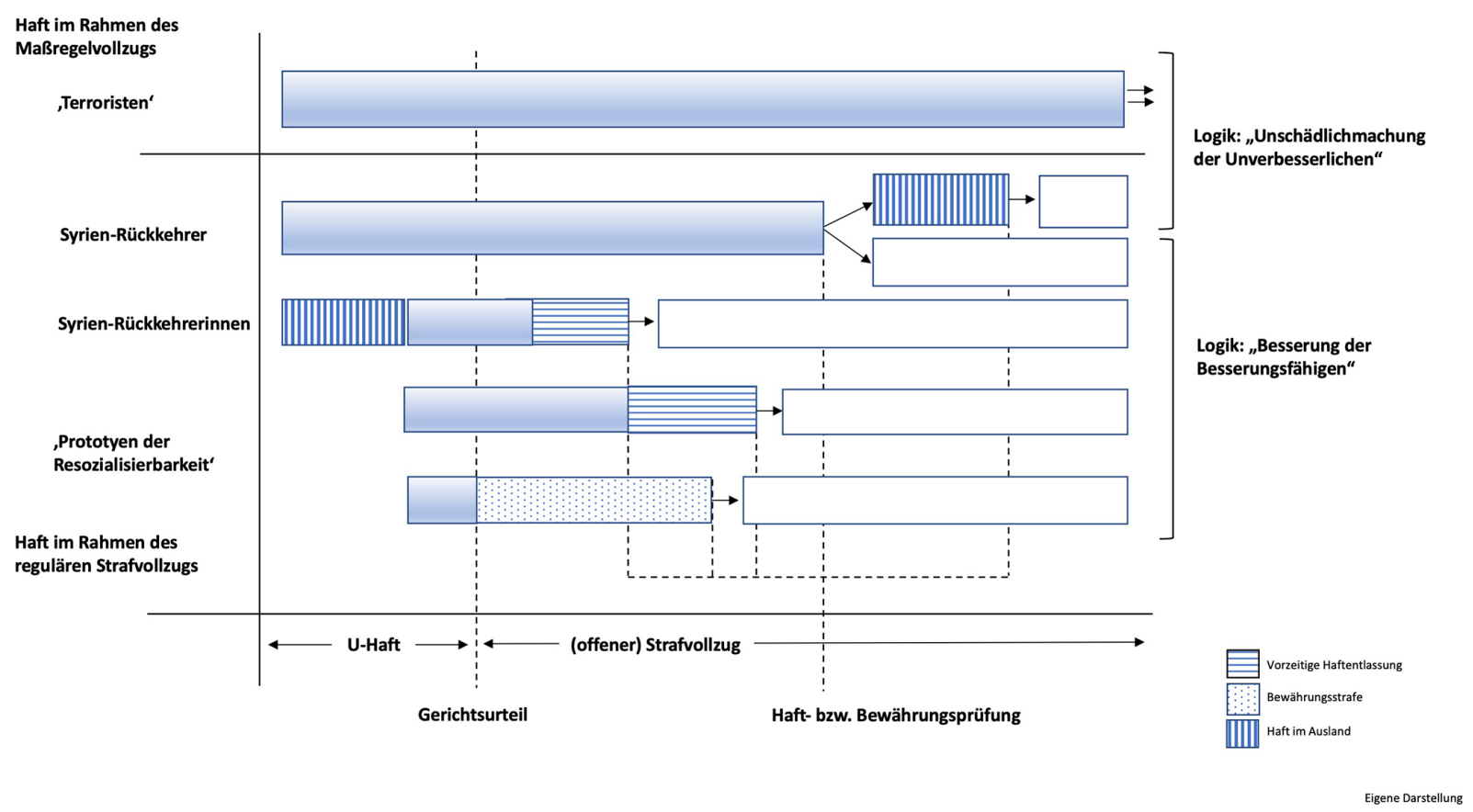

Abb. 5. Haftdauer und Verwahrungslogiken von am syrischen Bürgerkrieg beteiligter Personen deutscher wie ausländischer Staatsbürgerschaft der Jahre 2015-2020.

gen, Prozessverläufe und der in ihren Kontext eingebetteten Polizier- und Verwahrstatistiken der Jahre 2015 bis 2020 zeigt: Haft- und Sanktionsbestimmungen werden in und durch diese Prozesse in mehrfacher Hinsicht relational verhandelt, bewertet und verfügt - je nach richterlicher Lesart der zur Bewertung Vorgeführten. Diese Relationalität unterschiedlicher Härten für vergleichbare Straftaten zeigt sich in besonderer Weise in den in anderen Verfahrenskontexten als ,Kronzeugen“ Bezeichneten. Sie bilden hier den ersten Grundpfeiler für den Vergleich von Haftumständen, die aus eben solchen Strafverfahren resultierten (vgl. Abb. 5). Wie an anderer Stelle bereits ausführlicher dargestellt, sind es gerade diese Kronzeug:innen, die sich als „Prototypen der Resozialisierbarkeit“ (Klosterkamp und Reuber, 2017:265) präsentieren: Sie antworten, was von ihnen erwartet wird, nennen bereitwillig dringend benötigte Personen- und Situationsbeschreibungen und präsentieren sich dezidiert ,westlich ' gekleidet und kooperativ. Dies tun sie auch deshalb, um sich ihr Verhalten entlang der Achsen richterlicher Erwartungen durch die Organe der Rechtsprechung vergüten zu lassen nämlich mit verkürzten Haftdauern (vgl. ebd., Abb. 5). Wo die Gesetzgebung ein Strafmaß zwischen ein und zehn Jahren für die mitgliedschaftliche Beteiligung und Unterstützung von Vereinigungen, die dem terroristischen Spektrum gemäß $\S 129 \mathrm{a}+\mathrm{b}$ des StGB zugeordnet werden können, vorsieht, erhielten alle der insgesamt elf sich umfänglich einlassenden Angeklagten deutscher* Staatsbürgerschaft nicht mehr als fünf Jahre. Dies geschieht trotz des Umstandes, dass das Wissen um Ziele, Ressourcen und Routinen der
Vereinigungen nur durch die besonders lange, intensive (eigene) Einbindung in Geheimoperationen, Anschlagsplanungen oder Ausbildungsstätten durch die jeweiligen Preisgeber:innen hervorgebracht werden kann.

Diese verschiedenen Wissensbestände um individuelle Unterstützungs- und Kampfhandlungen stießen im Nachgang weitere Strafverfolgungen an. Sie wirkten sich strafverschärfend für die neu Angeklagten und strafmildernd für die Überbringenden aus. Gerade Letztere erhielten für ihre eigenen Strafprozesse gänzlich neue Prozessvorzeichen. So wurden bereits während ihrer Untersuchungshaft, spätestens aber zu Prozessbeginn die Trennscheibenverordnung fallen gelassen, die Prozesse selbst mit einer Länge von zwei bis zehn Verhandlungstagen erheblich verkürzt und auf ein Vorführen der Angeklagten in Hand- und Fußfesseln meist gänzlich verzichtet. Diese besonderen Lockerungen im Ablauf der Verhandlungen pausten sich auch auf die jeweiligen Haft- und Verwahrungsbedingungen durch, wie die jeweiligen Strafsenate offen verbalisierten: Zwei der 46 Angeklagten wurden unmittelbar nach der Urteilsverkündung mit einer Bewährungsstrafe und mindestens fünf unter Auflagen in die Freiheit entlassen. Für alle ,regulär' Verurteilten bedeutete die gesteigerte Kooperationsbereitschaft in den meisten Fällen zumindest eine Hafterleichterung. Unter Anwendung der ,2/3 Regelung ' wurden mindestens sechs von ihnen mit der Begründung der, guten Führung' frühzeitig entlassen. Gute Führung bedeutete hier die persönlich-individuelle Einpassung in die Logiken der Strafhaft und damit die Aufgabe westlich ausgedeuteter, ,radikal-islamistischer' Religi- 
onspraktiken (H1, 5. Juli 2016:9; D7, 3. November 2016) und der Verweis auf den bewussten Verzicht salafistischkonnotierter Kleidung und daraus abgeleiteten ,positiven Resozialisierungsprognosen“ (D10, 6. April 2017:1).

Diese Strafmilderungen betrafen vor allem eine Vielzahl der männlich-gelesenen Angeklagten der ersten Anklagewelle, die häufig die deutsche und in wenigen Fällen noch eine weitere Staatsangehörigkeit besaßen. Darüber hinaus traten sie zu einem Großteil dezidiert in denjenigen Verfahren ein, die sich gegen weibliche Angeklagte richteten. Viele dieser Angeklagten stützten in ihren Aussagen das auch in westlichen Konstruktionen um ,islamistischen Terrorismus " vorhandene Image der eher ,passiven Frauen'. Sie zeigten sich in ihren Eigenbeschreibungen des Grades an Beteiligungshandlungen vor allem als diejenigen, die für Reproduktions- und Sorgearbeiten zuständig waren. Somit wurden sie in und durch diese Prozesse und die ihnen vorgeschalteten Ermittlungen tendenziell häufiger als Opfer orientalistisch gerahmter patriarchaler $\mathrm{Zu}$ stände gelesen, denn als pönalisierungsbedürftige Rechtssubjekte skizziert. Dies war der Fall, obwohl sie zumindest in Teilen der Beispiele durchaus stärker in Kampf-, Propaganda- und Logistiktätigkeiten eingebunden waren als die mit ihnen assoziierten Männer (vgl. bspw. Klosterkamp, 2020:7; sowie für den britischen Kontext: Sjoberg und Gentry, 2016). Entlang der Feststellungen, inwiefern tägliche Gebete in der Haft verrichtet (M9, 9. September 2019:12), eine Gesichtsbedeckung während der Verhandlungen getragen (M10, 31. März 2017:42) und Kleinkinder von ihnen betreut werden (M11, 6. April 2017:132), fanden hier vor allem das ,Nachtatverhalten“ sowie vergeschlechtlichte Rollenzuschreibungen Eingang in entsprechende Urteilsbegründungen ${ }^{7}$. Diese Urteilsbegründungen zielten dabei nicht nur auf eigene Beteiligungshandlungen, sondern vor allem auf daraus abzuleitende Resozialisierungsprognosen ab. Viele machten darüber hinaus besondere Umstände in anderen, vorwiegend außereuropäischen Verwahr- und Transitlagern (vor allem der Türkei und dem Irak) geltend. Sie führten sowohl vor dem Oberlandesgericht Stuttgart als auch in Düsseldorf für alle weiblichen Angeklagten zu einer zweibzw. dreifachen Verrechnung mit der insgesamt abzuleistenden Freiheitsstrafe. In den meisten Fällen bedeuteten sie die vorzeitige Entlassung aus dem deutschen Vollzug (vgl. Abb. 5).

Wie diese Beispiele zeigen, ist somit im Kontext der ,Kriminologie des Selbst" (Garland, 2008:253) die Kooperationsbereitschaft während der Untersuchungshaft im Allgemeinen und speziell die Manövrier- und Passfähigkeit im Kontext strafmildernder Performanzen und Selbstanrufungen von Angeklagten richtungsgebend für die Verwahrungsdauer im deutschen Rechtssystem. Haft wird hier zu einem relational verhandelbaren Gut - sowohl für diejenigen, die

\footnotetext{
${ }^{7}$ Die Alterspanne der weiblichen Angeklagten belief sich auf 17 bis 48, die der männlichen auf 18 bis 64 .
}

sich der hiesigen Rechtsordnung in besonderer Weise als ,deradikalisiert', ,geläutert' und ,unterstützend ' präsentieren, als auch in der stärkeren Gewichtung ausländischer Haftumstände und daraus resultierender Hafterleichterungen im ,eigenen', ,milderen' Strafvollzugssystem. Andererseits wurde die Androhung und Durchsetzung von Haft als Mittel der Prävention und Abschreckungsinstrument gegen weitere Taten herangezogen ${ }^{8}$. Dabei wurde das Strafziel der Resozialisierung - punitiv wie diskursiv als ,Besserung der Besserungsfähigen “ des ,Eigenen “ - in den Vordergrund gerückt, im Gegensatz zu anderen Kontexten staatlicher Gefahrenabwehr (vgl. Schlepper, 2014:169; Naucke, 2010; Dübgen und Mattutat, 2017).

\section{2 ,Verwahrung der Unverbesserlichen“ - Haft unter den Vorzeichen der Sicherungsverwahrung}

Während die Verfahren der ersten und dritten Welle, die sich vorrangig gegen deutsche* Staatsbürger:innen richteten, in den meisten Fällen zu einer Relativierung der Haftund Strafverfolgungsumstände im Zuge glaubhafter Einlassungen und Läuterungsbekenntnisse führten, waren es gerade die Verfahren der hier konstatierten ,zweiten Welle ausländischer Staatsbürger, die den Rechtsstaat in besonderer Weise herausforderten. Im Zuge dieser Verfahren und unter Zuhilfenahme multipler Simultandolmetscherdienste wurde der Austausch über (geo-)politische Auseinandersetzungen und (geo-)kulturell aufgeladene Praktiken der Glaubensauslegung zu einer der entscheidenden Kernfragen nach Resozialisierungstendenzen und anzuleitender Verwahrungsform. Während im Fall vereitelter Anschläge in Düsseldorf und Köln die Hauptangeklagten mit sieben bzw. zehn Jahren Strafvollzug am oberen Ende des Spektrums sanktioniert und freiheitsentziehend verwahrt wurden, führten vor allem der gerichtlich aufgearbeitete Anschlag in einer EdekaFiliale in Hamburg-Harburg sowie ein erstes Verfahren gegen einen nach Deutschland geflüchteten Befehlshaber einer Untergruppierung der Freien Syrischen Armee zu einer besonderen Verwahrung im Zuge einer staatlich-induzierten Gefahrenabwehr.

Sich den Regeln und Ritualen des Gerichts während der Verhandlungen nur widerwillig fügend (D14, 13. Juni 2018:1; D17, 27. März 2020:1) und ohne einen Hinweis auf Reue oder partielle Zugeständnisse von Schuld, wurden ihre Taten am Ende in und durch die Arenen des Gerichts sowohl religiös-fundamentalistisch essenzialisiert

\footnotetext{
${ }^{8}$ So wurde etwa die Verurteilung eines jungen deutschen Mannes, der mehrmals bei seinem Versuch der Ausreise im Schengenraum gestoppt wurde und für eben jene Versuche dieselbe Haftstrafe erhielt wie die meisten derjenigen, die sich mehrere Monate im syrischen Kriegsgebiet aufhielten, als ,Pilot-Verfahren“ (M2, 9.Juli 2016:322) betitelt, das im Jahre 2016 ,den Horizont der Strafverfolgung erstmalig weit ins Vorfeld etwaiger Taten verlagerte (M14, 14. Juni 2016:1) und damit bereits den Versuch genauso ahndet wie die Ausreise selbst.
} 
und strafverstetigend pathologisiert. Unter Hinzunahme forensischer Gutachten und mit dem Verweis auf die ,besondere Schwere' der jeweiligen Ereignisse kam es im $\mathrm{Zu}$ ge der Verurteilungen zu einer Ausweitung der jeweiligen Höchstgrenzen (H2, 1. März 2018:2; D12, 24. September 2018:1). In anderen Fällen nichtdeutscher* Täterschaft - und hier vor allem im Zusammenhang mit, ausreisepflichtigen' bzw. lediglich ,geduldeten' Personen (D1, 12. August 2018; D4, 6. April 2017; S1, 6. Oktober 2016) - wurde eine Verbringung in ausländische Haftanstalten und/oder Abschiebung im Zuge kontinuierlicher Haftprüfungen erwirkt (vgl. Abb. 5). Diese Beispiele stehen stellvertretend für eine Reihe weiterer Fälle, die zumindest für die Jahre 2017 und 2018 mit 103 durchgeführten Abschiebungen beziffert werden können und von denen mindestens sechs Personen Verwahrungsumstände im Zusammenhang mit deutschen Staatschutzverfahren erfuhren (vgl. BT-Drucksache 19/5648, 2018:8; BT-Drucksache 19/15668, 2019).

Im Sinne einer ,Kriminologie der Anderen“ (Garland, 2008:253) und analog zu den von Korf konstatierten „Geographien des Ernstfalls“, nach denen „Sicherheit [... ] im Eigenen gefunden und im Containerstaat eingehegt, umgrenzt und abgeschottet [wird]“ (Korf, 2009:152), wurden hier die Bedrohungen, die ,von gefährlichen Orten außerhalb Europas oder von gefährlichen Elementen innerhalb unserer eigenen Gesellschaft, die außerhalb unserer Gemeinschaft [stehen] (ebd.), durch den Einsatz des Strafvollzugs voneinander getrennt. Und dies im Sinne eines „Governing through Crime“ (Simon, 2007) mit einem doppelt wirksamen Effekt: Während die ,Unverbesserlichen “ durch ihre Bewertung in medizinisch-wissenschaftlichen Kategorien ,kostengünstig ${ }^{9}{ }^{9}$, da dauerhaft, verwahrt wurden, erfolgte durch den Entzug von Bleiberechten zugleich auch eine Regulation staatschutzrechtlicher Devianz nichtdeutscher* Täter:innen. Hierbei gelten Ausweisungen bzw. dauerhafte Verwahrungen „nicht als Strafe im rechtlichen Sinn, sondern als Gefahrenabwehr" (Dübgen und Mattutat, 2017:91; siehe auch Graebsch, 2018). Dies wird besonders im Vergleich der ihnen entgegengestellten ,Besserungsfähigen “ deutlich. Deutschen* Staatsbürger:innen, die in die ,Ferne“ zogen und in das ,Eigene“ zurückkamen, wurden temporär von der Gesellschaft ausgeschlossen, aber ihnen wurde ,Besserung ' $\mathrm{zu}$ gestanden (vgl. Abschnitt 4.1). In den Fällen, in denen das ,Fremde“ ins ,Eigene' kam und die innere Sicherheit zu gefährden drohte, wurde jedoch eine Erweiterung des Strafrahmens angestrebt. Gleichzeitig wurde eine damit verbundene, dauerhafte Verwahrung oder Externalisierung dieser Personen eingesetzt und entsprechend legitimiert. Die Absonderung und Klassifizierung derjenigen, die als nicht (re-

\footnotetext{
${ }^{9}$,Kostengünstig ' meint hier ein Management im Sinne der,New Penology', das darauf ausgerichtet ist, ,to identify, classify, and manage groupings sorted by dangerousness' (Simon und Feeley, 1992:452). Grundlegend erweitert und modifiziert wurde die ,New Penology`als Gesellschaftsdiagnose von Simon (2007).
}

)integrierbar gelten, erfolgte dabei letztendlich unter Hinzunahme weiterer Rechtsgrundlagen (wie etwa $\S 58$ des Aufenthaltsgesetzes (AufenthG) oder $\S 63, \S 64$ StGB), die eine Beteiligung oder Mitwirkung an terroristischen Vereinigungen längst nicht mehr zum Gegenstand haben. So bestätigen diese empirischen Befunde nicht nur, was Garland 2008 als ,Geschichte der Gegenwart' für gesellschaftliche Institutionen der Kontrolle und Verwahrung im Allgemeinen konstatiert, nämlich dass ,das Gefängnis wieder zu einem Mittel des entmündigenden Freiheitsentzugs [...] [und] der Schutz der Bevölkerung zum bestimmenden Thema der Strafrechtspolitik geworden ist" (Garland, 2008:41). Sondern sie verdeutlichen auch darüber hinaus, inwiefern ,Strafen, die höher liegen als aus retributiven Erwägungen zu rechtfertigen wäre, [...] [wieder] möglich und sogar verpflichtend [werden]“ (ebd.). Und dies, wie die hier präsentierten Beispiele zeigen, umso mehr, je ,fremder' die Taten und ihre Entstehungskontexte zur ,eigenen ' Wirklichkeit stehen. Er sollte also Recht behalten.

\section{Fazit}

Im Bereich staatsschutzrelevanter Verfahren im Allgemeinen sowie in der Kontrolle, Sanktionierung und Verwahrung sog., islamistischer Terrorist:innen “ im Besonderen lässt sich gegenwärtig die Entstehung eines neu gestalteten Feldes der Verbrechenskontrolle und Strafjustiz beobachten. Dieses Feld fördert in der Art und Weise, ,wie' und ,wo' diese Prozesse geführt werden, welche Fragen gestellt werden und welche Körper als ,resozialisierbar" aus ihnen hervorgehen dürfen, in besonderer Weise eine dauerhafte Wegsperrung oder legitimierte Abschiebung delinquenter ,Fremder" sowie im Kontrast dazu, eine rechtliche wie verwahrungspraktische Bevorteilung des ,Eigenen ‘ zutage. So argumentiert dieser Beitrag, dass bei der Beurteilung von Taten im Kontext des ,islamistischen Terrorismus' kein einheitliches Konzept der Gefahrenabwehr und -bewältigung vorliegt, sondern vielmehr in einem multiplen Schnittfeld von ,Geschlecht', „Herkunft' und einer als ,religiös', ,fundamental ‘ und/oder ,politisch' ausgedeuteten (richterlichen) Analyse bestimmter Körper(-Praktiken) Anwendung findet. Dieser Umstand führt zu unterschiedlichen freiheitsentziehenden Maßnahmen, Vor- und Nachbeurteilungen oder eben Pathologisierungen. Er operiert dabei entlang der Logik ,Besserung der Besserungsfähigen“ vs. ,Verwahrung der Unverbesserlichen'. Demnach gilt der:die, islamistische Terrorist:in ' mal als gesamtgesellschaftliche Bedrohung existenziellen Ausmaßes, die entsprechende punitive Ausnahmemaßnahmen begründet, mal lediglich als ,ideologisch ' eingefärbte:r, aber prinzipiell (re-)integrierbare:r Kriminelle:r, um ihm:ihr die politische Bedeutung abzusprechen, den üblichen Strafrechtskatalog anzuwenden und eine Resozialisierung einleiten zu können. Neben der sicherheitsbetonten Bauweise, der in ihr zur Verhandlung vorgeführten, unterschiedlich positionierfä- 
higen Körper sowie den hier wirksamen diskursiv-normativ aufgeladenen (Un-)Sagbarkeiten sind dies eben jene Elemente der von Foucault konstatierten Logik staatlicher Strafund Vollzugsanstalten (Foucault, 1994 [1976]:38, 40, 178; 1981 [1969]:82), die im Sinne Garlands ,Kultur der Kontrolle' eine ,Normalisierung ' oder ,dauerhafte Verwahrung ' von delinquenten,Anderen ' im System zu begründen, zu verwehren oder in besonderer Weise zu begünstigen scheinen (Garland, 2008:253).

Angeleitet und in besonderer Weise legitimiert durch eine normativ-diskursive Risikoorientierung auf Verbrechen im Kontext einer als genuin männlich-migrantisch definierten Bedrohungslage, zeigt sich in und durch den systematischen Vergleich entsprechender Anklageschriften, Verfahrensabläufe und daraus abgeleiteter Verwahrungsumstände, inwiefern die Wertigkeit von Sicherheit und das Mittel der Strafhaft auch in politischen Verfahren wie denen des Staatsschutzes an Bedeutung gewinnt, in deren Zusammenhang verstetigte Haft oder gezielte Abschiebungen zum Garant eines Sicherheitsversprechens avancieren können. So gilt es, die Institution Gericht in ähnlicher Weise wie die Institution Gefängnis ,als einen gesellschaftlichen Ort von sozial segregierenden und differenzierenden Dynamiken und Prozessen zu begreifen, der nicht Lösung, Antwort oder Reaktion auf Kriminalität [oder eine staats- oder gesellschaftsbezogene Bedrohung ist], sondern den vielleicht zentralen Mechanismus ihrer Reproduktion darstellt" (Il-Tschung et al., 2017:74).

Durch die Verschneidung geographisch-ethnographischer Arbeiten mit einer feministisch-intersektionalen Analyse bundesdeutscher Polizier-, Sanktionier- und Verwahrpraktiken kann in Erweiterung bisheriger sozialwissenschaftlicher wie humangeographischer Arbeiten aufgezeigt werden, inwiefern in den Arenen rechtsprechender Praxis ein Abwägen von rassifizierenden und geschlechtsbasierten Sicherheitsund Risikoargumenten zur Urteilsbegründung und Legitimierung dauerhafter Verwahr- und Externalisierumstände in multiplen, doch stets machtgeladenen Herstellungs- und Subjektivierungsweisen punitiv Anwendung findet. Der Beitrag bietet somit auf einer theoretisch-konzeptionellen Ebene wertvolle Anknüpfungspunkte für eine weitere Auseinandersetzung mit institutionellen wie gesellschaftlichen Machtverhältnissen und zeigt darüber hinaus nicht zuletzt auch ernstzunehmende Handlungsbedarfe für eine rechtsprechende wie polizierende Praxis auf.

Datenverfügbarkeit. Die diesem Artikel zugrunde liegenden ethnographisch erhobenen Daten sind im Geographischen Institut der Universität Bonn archiviert und können auf Anfrage (und bei begrúndetem Interesse) eingesehen werden. Eine Herausgabe oder Veröffentlichung ist aufgrund von Datenschutzbestimmungen und dem Schutz von Persönlichkeitsrechten nicht möglich. Alle anderen Daten sind über das Archiv des Generalbundesanwalts (https: //www.generalbundesanwalt.de/DE/Home/home_node.html, letzter
Zugriff: 6. Mai 2021) (Generalbundesanwalt, 2021) oder über die Seite des Deutschen Bundestages (https://www.bundestag.de/ drucksachen, letzter Zugriff: 6. Mai 2021) (Bundestag, 2021) öffentlich zugänglich.

Interessenkonflikt. Die Autorin erklärt, dass kein Interessenkonflikt besteht.

Danksagung. Danken möchte ich vor allem Shadia Husseini de Araújo, Philipp Hövel und Silke Leibner für wertvolle Rückmeldungen zu verschiedenen Stadien dieses Textes sowie Lissa Peters und Josephine Fahr für ihre Unterstützung bei der Gestaltung von Grafiken und Recherchen. Benedikt Korf sowie zwei anonymen Gutachter:innen gilt mein Dank für inhaltlich weiterfúhrende Kritikpunkte und Empfehlungen, durch die mein Text im Laufe des Begutachtungsprozesses an Schärfe gewinnen durfte. Für die finanzielle Unterstützung danke ich der Fritz Thyssen Stiftung, die dadurch einen Teil der Forschung erst ermöglicht hat.

Finanzierung. Diese Forschung wurde durch die Fritz Thyssen Stiftung unterstützt (Fördernummer 10.18.2.008SO).

Begutachtung. This paper was edited by Benedikt Korf and reviewed by two anonymous referees.

\section{Literatur}

Agamben, G.: Homo sacer. Die souveräne Macht und das nackte Leben, Suhrkamp, Frankfurt am Main, 2002.

Belina, B.: Kriminalitätskartierung - Produkt und Mittel neoliberalen Regierens, oder: Wenn falsche Abstraktionen durch die Macht der Karte praktisch wahr gemacht werden, Geogr. Z., 97, 192-212, 2009.

Belina, B.: Predictive Policing, Monatsschrif. Kriminol. Strafrechtsref., 99, 85-100, 2016.

Brazzell, M.: Transformative Gerechtigkeit statt Polizei und Gefängnisse: Für einen alternativen Umgang mit sexualisierter Gewalt und Beziehungsgewalt, in: Kritik der Polizei, Herausgeber: v. Loick, D., Campus, Frankfurt am Main, 279-298, 2018.

BT-Drucksache 19/5648: Personenpotentiale islamistischer ,Gefährder', Deutscher Bundestag, online aufrufbar: https:// dip21.bundestag.de/dip21/btd/19/056/1905648.pdf (letzter Zugriff: 6. Mai 2021), 2018.

BT-Drucksache 19/6684: Islamistischer Terrorismus in Deutschland, Deutscher Bundestag, online aufrufbar: https://dip21.bundestag.de/dip21/btd/19/066/1906684.pdf (letzter Zugriff: 6. Mai 2021), 2018.

BT-Drucksache 19/11907: Verfahren des Generalbundesanwalts beim Bundesgerichtshof seit dem Jahr 2013, Deutscher Bundestag, online aufrufbar: http://dipbt.bundestag.de/extrakt/ba/ WP19/2506/250651.html (letzter Zugriff: 6. Mai 2021), 2019.

BT-Drucksache 19/15668: Praxis der Abschiebung von Gefährdern, Deutscher Bundestag, online aufrufbar: http://dipbt. 
bundestag.de/extrakt/ba/WP19/2554/255460.html (letzter Zugriff: 6. Mai 2021), 2019.

BT-Drucksache 19/20201: IS-Anhänger und deren Kinder im Inund Ausland, Deutscher Bundestag, online aufrufbar: http:// dipbt.bundestag.de/extrakt/ba/WP19/2630/263059.html (letzter Zugriff: 6. Mai 2021), 2020.

Bundestag: Drucksachen, online aufrufbar: https://www.bundestag. de/drucksachen, letzter Zugriff: 6. Mai 2021.

Dübgen, F. und Mattutat, L.: Neoliberalisierung im Vollzug. Gibt es einen ,Prison-Industrial Complex ' in Deutschland?, WestEnd, 2, 77-98, 2017.

Egbert, S. und Krasmann, S.: Predictive Policing. Eine ethnographische Studie neuer Technologien zur Vorhersage von Straftaten und ihre Folgen für die polizeiliche Praxis, Projektabschlussbericht, Universität Hamburg, Hamburg, 2019.

Faria, C., Klosterkamp, S., Torres, R., und Walenta, J.: Embodied Exhibits: Toward a Feminist Geographic Courtroom Ethnography, Ann. Am. Assoc. Geogr., 110, 1195-1113, 2020.

Foucault, M.: Dispositive der Macht, Merve Verlag, Berlin, 1978.

Foucault, M.: Archäologie des Wissens, Suhrkamp, Frankfurt am Main, [1969] 1981.

Foucault, M.: Überwachen und Strafen: Die Geburt des Gefängnisses, Suhrkamp, Frankfurt am Main, [1976] 1994.

Foucault, M.: Geschichte der Gouvernementalität I: Sicherheit, Territorium, Bevölkerung, in: Vorlesung am Collège de France, Herausgeber: v. Sennelart, M., Suhrkamp, Frankfurt am Main, [1977-78] 2004.

Garland, D.: Kulturen der Kontrolle, Campus, Frankfurt am Main, 2008.

Generalbundesanwalt: „Unser Rechtsstaat braucht Einsatz und Vertrauen!“, online aufrufbar: https://www.generalbundesanwalt.de/ DE/Home/home_node.html, letzter Zugriff: 6. Mai 2021.

Gericke, B. und Kallert, T.: Zum Outcome der Maßregelvollzugsbehandlung nach § 64 StGB, Psychiatrische Praxis, 34, 218-226, 2007.

Gilmore, R. W.: Golden Gulag, University of California Press, Berkeley, 2007.

Graebsch, C.: Resozialisierung bei nichtdeutscher Staatsangehörigkeit, in: Resozialisierung: Handbuch, 4. Auflage, Herausgeber: v. Cornel, H., Kawamura-Reindl, G., und Sonnen, B. R., Nomos, Baden-Baden, 433-448, 2018.

Hannah, M.: Torture and the Ticking Bomb: The War on Terrorism as a Geographical Imagination of Power/Knowledge, Ann. Assoc. Am. Geogr., 96, 622-640, 2006.

Herbert, S.: Too Easy to Keep, University of California Press, Oakland, 2019.

Il-Tschung, L., Loick, D., Marquardt, N., und Trautmann, F.: Delinquenzmilieu. Armut und Gefängnis am Beispiel der Ersatzfreiheitsstrafe, WestEnd, 2, 125-137, 2017.

Jeffrey, A.: Legal geography 1: Court materiality, Prog. Human Geogr., 43, 565-573, https://doi.org/10.1177/0309132517747746, 2019.

Keitzel, S.: Varianzen der Verselbstständigung der Polizei per Gesetz. Gefährliche Orte im bundesweiten Vergleich, Kriminolog. J., 3, 191-209, 2020.

Klosterkamp, S.: Security, Mobility, and the Body - Syrian insurgent groups' infrastructures and their geopolitical contestations through/by/in legal institutions, Polit. Geogr., 84, 102301, https://doi.org/10.1016/j.polgeo.2020.102301, 2020.
Klosterkamp, S. and Reuber, P.: „Im Namen der Sicherheit“ - Staatsschutzprozesse als Orte politisch-geographischer Forschung, dargestellt an Beispielen aus Gerichtsverfahren gegen Kämpfer und UnterstützerInnen der Terrororganisation „Islamischer Staat“, Geogr. Helv., 72, 255-269, https://doi.org/10.5194/gh-72-255-2017, 2017.

Korf, B.: Geographie des Ernstfalls, Geogr. Z., 97, 151-167, 2009.

Krasmann, S.: Der Feind an den Grenzen des Rechtsstaats, in: Foucault: Diskursanalyse der Politik. Eine Einführung, Herausgeber: v. Kerchner, B. und Schneider, S., VS Verlag für Sozialwissenschaften, Wiesbaden, 233-250, 2006.

Loick, D. (Hrsg.): Kritik der Polizei, Campus, Frankfurt am Main, 2018.

Marquardt, N.: Armut und Gefängnis - Kritische Perspektiven auf den Zusammenhang von Verschuldung und Freiheitsentzug, Geographisches Kolloquium, Münster, 30. April 2019.

Munk, T. B.: 100,000 false positives for every real terrorist Why anti-terror algorithms don't work, online aufrufbar: https:// firstmonday.org/ojs/index.php/fm/article/view/7126 (letzter Zugriff: 6. Mai 2021), 2017.

Naucke, W.: Die robuste Tradition des Sicherheitsstrafrechts, Kritische Vierteljahresschrift für Gesetzgebung und Rechtswissenschaft (KritV), 93, 129-136, 2010.

Pain, R.: Globalized fear? Towards an emotional geopolitics, Prog. Human Geogr., 33, 466-486, 2009.

Paulsen, A.: 100 Jahre Oberlandesgericht Düsseldorf, Berliner Wissenschafts-Verlag, Berlin, 2006.

Reuber, P. und Strüver, A.: Der Anschlag von New York und der Krieg gegen Afghanistan in den Medien - Eine Analyse der geopolitischen Diskurse, in: Die Politik räumlicher Repräsentation, Herausgeber: v. Dzudzek, I., Reuber, P. und Strü ver, A., Forum Politische Geographie, Berlin, Münster, 179-216, 2012.

Rhodes, L. A.: Madness and Reason in the Maximum Security Prison, University of California Press, Berkeley, 2004.

Richter, M., Hostettler, U., und Martin, I.: Chronik eines angekündigten Todes: Trajektorien und Logiken am Lebensende im Schweizer Strafvollzug, Schweiz. Z. Kriminol., 1, 21-30, 2017.

Rodriguez, F., Curry, T., und Lee, G.: Gender Differences in Criminal Sentencing: Do Effects Vary Across Violent, Property, and Drug Offenses?, Social Sci. Quart., 87, 318-39, 2006.

Schlepper, C.: Strafgesetzgebung in der Spätmoderne, Springer Verlag, Wiesbaden, 2014.

Schliehe, A.: ,Inside the Carceral': Girls and Young Women in the Scottish Criminal Justice System, Scottish Geogr. J., 130, 71-85, 2014.

Simon, J.: Governing Through Crime: How the War on Crime Transformed American Democracy and Created a Culture of Fear, Oxford University Press, Oxford, 2007.

Simon, J. und Feeley, M. M.: The New Penology: Notes on the Emerging Strategy of Corrections and Its Implications, Criminology, 30, 449-474, 1992.

Singelnstein, T.: Confirmation Bias - Die Bestätigungsneigung als kognitive Verzerrung bei polizeilichen Ermittlungen im Strafverfahren, Strafverteidiger, 36, 830-836, 2016.

Sjoberg, L. und Gentry, C. E.: It's Complicated: Looking Closely at Women in Violent Extremism, Georgetown J. Int. Affair., 17, 23-30, 2016. 
Turner, J.: Introduction: Criminality and carcerality across boundaries, Geogr. Helv., 69, 321-323, https://doi.org/10.5194/gh-69321-2014, 2014.
Zöller, M. A.: Strafrechtliche Verfolgung von Terrorismus und politischem Extremismus unter dem Einfluss des Rechts der Europäischen Union, Zeitschrift für internationale Strafrechtsdogmatik, 9, 402-411, 2014. 